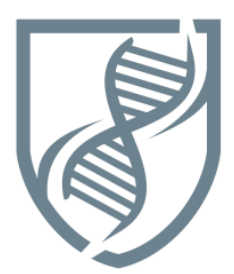

Journal of Bioscience and Applied Research

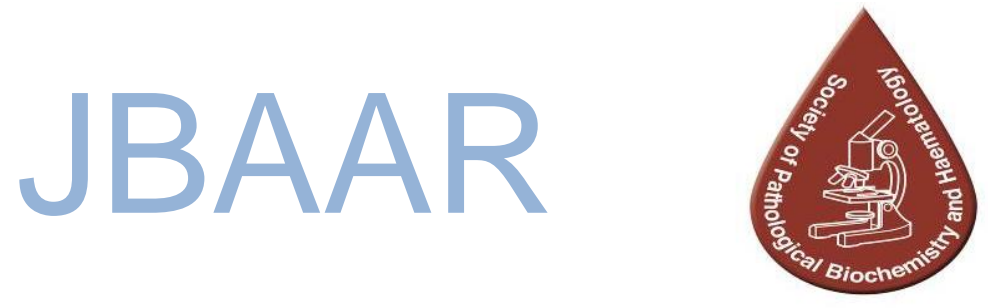

WWW.JBAAR.ORG

\title{
Evaluation of Nuclepore Membrane Filtration Technique for Diagnosis of Schistosoma haematobium Infection
}

\author{
Ahmed Abdelhalim Yameny \\ Society of Pathological Biochemistry and Hematology, Egypt \\ Ahmed A. Yameny (Email: dr.ahmedyameny@yahoo.com) \\ DOI: $10.21608 /$ jbaar.2015.106044
}

\begin{abstract}
Schistosomiasis is a parasitic disease. It is one of the most important diseases of humans in tropical and subtropical parts of the world, rapid characterization of schistosome eggs considered a key step for clinical management, diagnosis of Schistosoma haematobium is generally based on the detection of eggs in a single urine specimen, Urine filtration is one of the methods recommended by the WHO for the detection of S.haematobium. In 1976, Peters et al adapted a nuclepore filter for the diagnosis of S.haematobium eggs. They used a filter $13 \mathrm{~mm}$ in diameter with $8 \mu \mathrm{m}$ pores, in another study done by Peters et al (1976), they proved that under field conditions, filtration of two $5 \mathrm{ml}$ aliquots using $8 \mu \mathrm{m}$ pore filters was very practical, if eggs of S.haematobium are present (size 150 by $60 \mu \mathrm{m}$ ), they are unable to pass through the filter and can be observed and counted under a microscope fitted with a 10x objective.

This study used both the microscopic examination techniques of urine, centrifugation sedimentation technique and Nuclepore membrane filtration technique to detect Schistosoma haematobium eggs in urine for 1000 patient samples, to evaluate each technique alone and the two techniques together. This study showed that the nuclepore filtration method more valid than the sedimentation centrifugation method, nuclepore filtration detect 77 infected cases with a sensitivity of $97.5 \%$, but sedimentation detect 64 infected cases with a sensitivity of $81 \%$, in this study we considered the sum of nuclepore and sedimentation as a gold standard, they detected 79 infected cases to compare them with other methods.
\end{abstract}

Keywords: Schistosoma haematobium, Nuclepore Membrane Filtration, Parasitological method, Technique, Schistosoma eggs 


\section{Introduction}

Schistosomiasis is a parasitic disease. It is one of the most important diseases of humans in tropical and subtropical parts of the world, being second to Malaria in public health and socioeconomic importance among parasitic diseases ${ }^{(1,2)}$. Schistosoma haematobium eggs are excreted into urine characterized by oval-shaped with a miracidium inside and external terminal spine ${ }^{(3)}$. Rapid characterization of schistosome eggs is considered a key step for clinical management, due to the low concentration of eggs and matrix effects of urine ${ }^{(4,5)}$. Diagnosis of S.haematobium is generally based on the detection of eggs in a single urine specimen, the excretion of eggs in urine is highest between 10:00h and 14:00 $\mathrm{h}$ with a peak around mid-day. Even when persons are heavily infected eggs may not be present in the urine all the time $^{(6)}$. Day-to-Day variation and circadian variation may then lead to false conclusions about the prevalence and incidence of the disease ${ }^{(7)}$. The quantitative diagnosis of S.haematobium has been based on the microscopic detection of Schistosome eggs in urine ${ }^{(8)}$. The level of egg output is thought to reflect the worm burden and to determine the severity of urinary tract disease ${ }^{(9,10)}$. The two methods used for the detection of S.haematobium ova are sedimentation and filtration. The sedimentation method is less sensitive but cheaper and simpler to perform ${ }^{(11)}$. If a known volume of sediment from a measured volume of urine $(10 \mathrm{ml})$ is examined, the number of eggs counted can be related to the total urine volume. However, egg output is usually expressed in terms of ova/10 ml urine ${ }^{(12) .}$ Urine filtration is one of the methods recommended by the WHO for the detection of S.haematobium ${ }^{(13)}$. In most quantitative techniques used for S.haematobium diagnosis, transparent nucleopore filters were available and described to be used in the diagnosis of filariasis by the WHO committee in $1974^{(14)}$. In 1976, Peters et al adapted a nuclepore filter for the diagnosis of S.haematobium eggs. They used a filter $13 \mathrm{~mm}$ in diameter with $8 \mu \mathrm{m}$ pores ${ }^{(15)}$. In another study done by Peters et al (1976), they proved that under field conditions, filtration of two $5 \mathrm{ml}$ aliquots using $8 \mu \mathrm{m}$ pore filters was very practical ${ }^{(16)}$. The filtration device is composed of a plastic filter holder that contains a nylon filter (pore size 12 to $20 \mu \mathrm{m}$ ). Complete filtration of the urine sample is ensured by a rubber O-ring that prevents urine from bypassing the filter. For diagnostic purposes, a standard $10 \mathrm{ml}$ quantity of the urine to be tested is forced through the device with a syringe. If eggs of S.haematobium are present (size 150 by $60 \mu \mathrm{m}$ ), they are unable to pass through the filter and can be observed and counted under a microscope fitted with a 10x objective ${ }^{(17)}$. There is no agreement concerning the possibility of filter reuse. For practical purposes, filter holders can be reused and also filters from negative tests after thorough washing. Filters from positive tests should be discarded ${ }^{(18,19)}$. The filtration technique is used in public health care mainly when quantitative information is required ${ }^{(11)}$. The present study used both the microscopic examination techniques of urine, centrifugation sedimentation technique and Nuclepore membrane filtration technique to detect Schistosoma haematobium eggs in urine for 1000 patient samples, to evaluate each technique alone and the two techniques together.

\section{Research objective :}

To evaluate the Nuclepore Membrane Filtration Technique for Diagnosis of Schistosoma haematobium Infection

\section{Material and methods}

\subsection{Study population and ethical consideration}

This study included 1000 patients attending the Ministry of health laboratory centers, in El-Fayoum Governorate, these study subjects were randomly selected irrespective of the age-group and both genders were included. All the studied populations were informed about the purpose of sample collection and their consents were obtained. Patients were free to refuse sample collection.

\subsection{Study design}

This research is a cross-sectional study designed to evaluate the Nuclepore filtration technique to determine urinary schistosomiasis in the study population. This study was targeting customers who came for laboratories of health centers for urine analysis. 


\subsection{Collection and processing of urine samples:}

Clean specimen bottles were labeled with the needed information and issued to the participating individuals whose informed consent was sought earlier, each patient was given a wide mouth screw-capped container into which to void urine. This was carried out between 10.00 am and $2.00 \mathrm{pm}$ when the ova count of S.haematobium is expected to be at its peak $^{(20)}$. In this study, urinary schistosomiasis was defined as the presence of ova of S.haematobium in the urine.

\subsection{Urine microscopy}

Urine examination was carried out in the laboratory of health centers and by the researcher. Urine samples were examined for the presence of S.haematobium eggs as in the sedimentation method of Cheesbrough (2006) (21). Each urine sample was mixed thoroughly with a glass rod and two samples were taken each $10 \mathrm{ml}$ urine, one sample for sedimentation centrifugation, and the other $10 \mathrm{ml}$ urine sample for the Nuclepore membrane filtration technique. The first $10 \mathrm{ml}$ transferred into a centrifuge tube and centrifuged at $2000 \mathrm{rpm}$ for 5 minutes at room temperature. The supernatant was then discarded and sediment was transferred to a microscope glass slide and covered with a coverslip. A drop of Lugol's Iodine was added onto the coverslip before the examination. The examination of the entire sediment was carried out using the $\mathrm{x} 10$ objective of a compound light microscope.

The second $10 \mathrm{ml}$ urine sample was examined using the Nuclepore membrane filtration technique for S.haematobium eggs detection as in the method of Cheesbrough (2009) ${ }^{(22)}$.

\subsection{Nuclepore Membrane Filtration Technique:}

1- For 1000 urine samples, a polycarbonate or nylon filter (pore size 12-20 $\mu \mathrm{m}$ ) was placed in the filter holder. A urine sample was agitated by shaking it gently or by filling and emptying the syringe twice.

2- $10 \mathrm{ml}$ of the urine was drawn into the syringe and the filter-holder was attached to the bottom of the syringe.

3- The filter holder was carefully unscrewed, and the air was drawn into the syringe. The syringe was reattached to the holder, and the air was expelled. This was important as it helped to remove excess urine and also made sure the eggs, if present, were attached to the filter

4- The filter holder was unscrewed, the filter was removed with the forceps and placed (top side up) on a microscope slide. one drop of Lugol's iodine was added and left for 15 seconds for the stain to penetrate the eggs.

5- The whole filter was examined under the microscope immediately at high power(40x). Schistosome eggs stain orange and can be seen clearly.

\subsection{Statistical analysis}

Results collected, coded, tabulated, and analyzed through computer facilities using statistical methods S.haematobium infection was defined as any number of eggs greater than zero found in $10 \mathrm{ml}$ of urine, was performed to evaluate the Nuclepore Membrane Filtration Technique for Diagnosis of Schistosoma haematobium Infection.

\section{Results}

Percentages of S.haematobium infection: Table ( 1$)$ shows percentages of S.haematobium infection as detected by different direct parasitologic techniques. The sedimentation technique revealed a percentage of $6.4 \%$ which increased to $7.7 \%$ using the nuclepore filtration technique. Prevalence increased to $7.9 \%$ when the results of both techniques were considered. Sedimentation with a sensitivity of $81 \%$ while nuclepore filtration sensitivity

was

$97.5 \%$ 
Table (1 ): Percentages of S.haematobium infection among the study sample according to urine examination by direct parasitologic techniques.

\begin{tabular}{|l|c|c|c|c|l|}
\hline \multirow{2}{*}{ Direct } & \multicolumn{4}{|c|}{ S.haematobium } & \multirow{2}{*}{ Parasitologic Techniques } \\
\cline { 2 - 5 } & \multicolumn{2}{|c|}{ Negative } & \multicolumn{2}{c|}{ Positive } & \multirow{2}{*}{ Total } \\
\cline { 2 - 5 } & NO. & $\%$ & NO. & $\%$ & \\
\hline Sedimentation & 936 & 93.6 & 64 & 6.4 & 1000 \\
\hline Nuclepore filtration & 923 & 92.3 & 77 & 7.7 & 1000 \\
\hline Both techniques & 921 & 92.1 & 79 & 7.9 & 1000 \\
\hline
\end{tabular}

\section{Sedimentation:}

Sensitivity $=81 \%$

Specificity $=100 \quad \%$

Positive predictive value $=100 \%$

Negative predictive value $=98.4 \%$

\section{Nuclepore filtration:}

Sensitivity $=97.5 \%$

Specificity $=100 \%$

Positive predictive value $=100 \%$

Negative predictive value $=99.78 \%$

(Figure1): Percentages of S.haematobium infection among study samples according to urine examination by direct parasitologic techniques.

\section{PARASITOLOGIC TECHNIQUES}

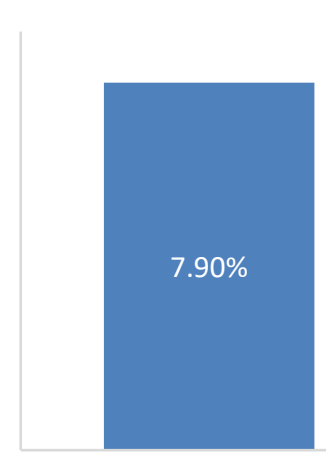

BОTH
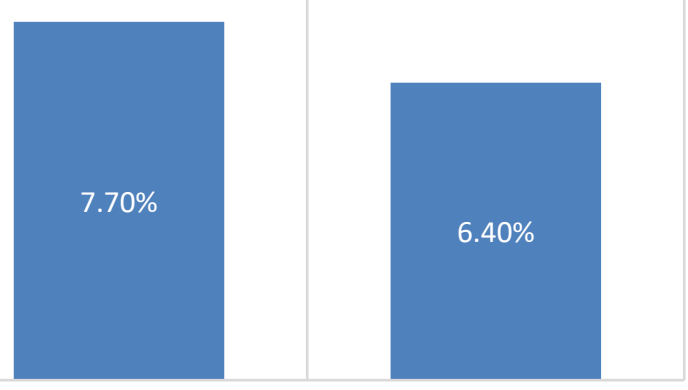


\section{Discussion}

Schistosomiasis is usually diagnosed by parasitological (microscopic detection of eggs) or immunological methods (presence of specific antibodies or circulating schistosomal antigens). The demonstration of eggs in urine or feces confirms the presence of adult worms, but failure to detect eggs, even after repeated examinations of urine or stool samples, does not exclude active schistosomiasis ${ }^{(24)}$.

\subsection{Parasitologic techniques}

This study showed that the nuclepore filtration method more valid than the sedimentation centrifugation method, nuclepore filtration detect 77 infected cases with a sensitivity of $97.5 \%$, but sedimentation detect 64 infected cases with a sensitivity of $81 \%$ in this study we considered the sum of nuclepore and sedimentation as a gold standard, they detected 79 infected cases to compare them with other methods.

Urine filtration is one of the methods recommended by the World Health Organization for the detection of schistosoma haematobium ${ }^{(9)}$. Parasitological diagnosis of schistosoma haematobium infection is readily undertaken by urine filtration. While urine is easily collected, due to the circadian pattern of egg excretion, specimens should ideally be collected between 10 am and 2 pm, and preferably after physical exercise ${ }^{(24)}$.

\subsection{Quantitative urine examination:}

Quantitative data from urine examination by the syringe filtration technique for detection of S. haematobium infection may be reported according to egg count categories:

Light infection -1-49 eggs per $10 \mathrm{ml}$ of urine.

Heavy infection -> 50 eggs per $10 \mathrm{ml}$ of urine ${ }^{(11)}$.

\section{Conclusion and Recommendations}

Urine filtration is one of the methods recommended by the World Health Organization for detection of schistosoma haematobium infection, This study showed that the nuclepore filtration method more valid than the sedimentation centrifugation method, nuclepore filtration detects 77 infected cases with a sensitivity of $97.5 \%$, but sedimentation detect 64 infected cases with a sensitivity of $81 \%$ in this study we recommended using the sum of nuclepore and sedimentation as a gold standard, they detected 79 infected cases to compare them with another method.

\section{Conflict of interest}

There are no conflicts of interest.

\section{Financial support and sponsorship}

This research did not receive any specific grant from funding agencies in the public, commercial, or not-for-profit sectors.

\section{References}

1. Barakat, R.M.R., 2013. Epidemiology of Schistosomiasis in Egypt: travel through time: a review. J. Adv. Res. 4 (5), 425-432. http://dx.doi.org/10.1016/ j.jare.2012.07.003.

2. Chitsulo, L., Engels, D., Montresor, A., Savioli, L., 2000. The global status of schistosomiasis and its control. Acta Trop. 77, 41-51.

3. Gryseels B, Polman K, Clerinx J, Kestens L. Human schistosomiasis. Lancet. 2006; 368(9541):1106-18. DOI: 10.1016/S0140-6736(06)69440-3 PMID: 16997665.

4. Chiu ML, Lawi W, Snyder ST, Wong PK, Liao JC, Gau V. Matrix Effects-A Challenge toward Automation of Molecular Analysis. Journal of the Association for Laboratory 
Automation. 2010; 15(3): 233-42. DOI: 10.1016/j.jala.2010.02.001

5. Mach KE, Wong PK, Liao JC. Biosensor diagnosis of urinary tract infections: a path to better treatment? Trends Pharmacol Sci. 2011; 32(6):330-6. DOI: 10.1016/j.tips.2011.03.001 PMID: 21458868; PubMed Central PMCID: PMCPMC3106133.

6. Cheesebrough M. district laboratory practice in tropical countries. Cambridge; 1999 (part 1); 236-239, 372-374.

7. Etten LV, Kremsner PG, Karigger FW, Deelder AM. Day-to-day variation of egg output and schistosome circulating antigens in urine of Schistosoma haematobium infected school children from Gabon and follow-up after chemotherapy. Am J Trop Med Hyg 1997; 57(3): 337-341.

8. Ndhlovu $\mathrm{P}$, Cadman $\mathrm{H}$, Gundersen $\mathrm{S}$, Vennervuld BJ, Friis H, Christensen NO, et al. Circulation anodic antigen (CAA) levels in different age groups in Zimbabwean rural community endemic for schistosoma haematobium determined using the magnetic beads antigen-capture enzyme-linked immunoassay. Am J Trop Hyg 1996; 54(5): 537-542.

9. - Poggensee G, Krartz I, Kiwelau I, Feldmeiern H. Screening of Tanzanian women of childbearing age for urinary schistosome: Validity of urine reagent strop reading and self. Reported Symptoms-Bulleting of the world health organization, 2000, 78 (4).

10. Forsyth DM, Macdonald G. Urological complications of endemic schistosomiasis in school children. Part 2- Donge school, Zanzibar. Trans R Soc Trop Med Hyg 1966; 60: 568-578.
11. World Health Organization. Basic Laboratory methods in medical parasitology, Geneva 1991; 33-36.

12. Davis A (1968) Bull World Health Org 38, 197.

13. World Health Organization. 1993. The control of schistosome second report of the WHO Expert Committee. World Health Organization, Geneva, Switzerland.

14. WHO expert committee on filariasis. WHO Tech Rep Ser 1974; 342: 54.

15. Peters PA, Warrenks, and Mahmoud AAF. Rapid accurate quantification of schistosome eggs via nucleopore filters. J Parasit 1976; 62 (1): 154-155.

16. Peters PA, Mahmoud AAF, Warren KS, Ouma JH, Arapsiongok TK. Field studies of a rapid, accurate means of quantifying schistosoma haematobium eggs in urine samples. Bull WHO 1976; 54: 159-162.

17. Gyorks TW, Ramsan M, Foum A, Khamis IS. Efficacy of new low-cost filtration device for recovering Schistosoma haematobium eggs from urine. Journal of Clinical microbiology 2001; 39(7): 2681-2682.

18. Mshinda H, Lengeler C, Hatz C, de Savigny D. field diagnosis of urinary schistosomiasis by multiple uses of nuclepore urine filters. J Parasitol 1989; 75: 476-478.

19. Rohde R, Abrawn R, Rasoloarison C. Potential false positive egg-counts through the revse of polyamide filters in the diagnosis of urinary schistosomiasis. Trop Med Parasitol 1985; 36: 143-144.

20. Eyong ME, Ikepeme EE, Ekanem EE. Relationship between Schistosoma haematobium infection and urinary tract infection among children in South Eastern, Nigeria. Niger Postgrad Med J2008;15:89-93.

21. Cheesbrough M. District laboratory practice in tropical countries part 2. United Kingdom: 
Cambridge University Press. Cambridge. 2006; $323-41$

22. Cheesbrough M. Laboratory-practice-intropical-countries-part-1. United Kingdom: Cambridge University Press. Cambridge. 2009;p.357

23. - WHO Report: Report of an informal working group meeting on urogenital schistosomiasis and HIV transmission Geneva, Switzerland, 1-2 October 2009.

24. Feldmeier H(1993) Diagnosis in human schistosomiasis .In : Jordan P,Webbe $\mathrm{G}$, Sturrock R, editors .Human schistosomiasis .Oxon: CAB International .pp.271- 303. 